\title{
Non-Invasive Respiratory Support During Covid-19 Pandemic
}

\author{
José Gr González García ${ }^{1^{*}}$, Oswaldo A Caguana ${ }^{1}$, Roberto Chalela ${ }^{1,3}$ and Joaquim Gea ${ }^{1-3}$ \\ ${ }^{1}$ Department of Respiratory Medicine, Hospital del Mar - IMIM, Spain \\ ${ }^{2}$ DCEXS, Pompeu Fabra University, Spain \\ ${ }^{3}$ CIBERES, ISCiii, Spain
}

*Corresponding author: José G González-García, Department of Respiratory Medicine, Spain

To Cite This Article: José G González-García. Non-Invasive Respiratory Support During Covid-19 Pandemic. 2020 - 10(3). AJBSR.MS.ID.001509. DOI: 10.34297/AJBSR.2020.10.001509.

Received: 恝 August 26, 2020; Published: 眥 September 17, 2020

\begin{abstract}
Acute respiratory failure frequently occurs in severe COVID-19 and requires ventilator support. However, during the first wave of the pandemic, conventional resources for critically ill patients were insufficient in many centers. Therefore, an alternative strategy based on the use of non-invasive respiratory support (NIRS), and especially continuous positive airway pressure (CPAP), have been massively employed, either as a definitive therapy or as a bridge for orotracheal intubation. The results of this strategy have been excellent. If patients are carefully selected and monitored and NIRS is managed by an expert team in an appropriate environment, the vast majority of patients survive, avoiding intubation in most cases, and even decreasing the possibility of a self-inflicted lung injury (SILI). Moreover, there is evidence that with these techniques the risk of aerosolization is low. Nowadays, CPAP is considered the best option for most patients, with BiPAP being especially recommended in those with previous thoracic diseases or chronic hypercapnia. We conclude that NIRS techniques can be used in the initial care of most patients with severe respiratory failure from COVID-19 pneumonia and/or thromboembolism. To achieve the best results, intermediate care units, where specialized respiratory support can be provided, are the most appropriate framework.
\end{abstract}

Keywords: Respiratory Failure; Respiratory Support; COVID-19; Intermediate Units

Abbreviations: CPAP: Continuous Positive Airway Pressure; NIRS: Non-Invasive Respiratory Support; SILI: Self-Inflicted Lung Injury; SARSCoV-2: Severe Acute Respiratory Syndrome-CoV-2; ARDS: Acute Respiratory Distress Syndrome; CPAP: Continuous Positive Airway Pressure; HFNC: High Flow Through Nasal Cannula; BiPAP: Two-Level Positive Airway Pressure, VA/Q: Ventilation/Perfusion Ratio

\section{Introduction}

Since the pandemic was declared in March 2020, severe acute respiratory syndrome due to coronavirus type 2 (SARS-CoV-2) infection has been a critical global health problem. The first cases were reported in late December in Wuhan city, capital of the Hubei Province in China [1]. The disease caused by this infection is known as COVID-19 and has currently produced more than 550,000 deaths in 200 countries despite the great efforts and strict quarantines in most of these countries [2]. Most cases are considered as a mild disease but nearly $14 \%$ require hospitalization, mostly for severe bilateral 'pneumonia' with oxygen requirements, and 5\% need critical care and invasive mechanical ventilation [3]. Patients with SARS-CoV-2 pneumonia may also develop severe Acute Respiratory Distress Syndrome (ARDS), as defined by Berlin criteria, during the clinical course of the disease [4]. This syndrome appears around the second week after the onset of symptoms. Furthermore, severe COVID-19 caused by bilateral involvement of the lung parenchyma can be seriously aggravated in many cases by the coexistence of pulmonary thromboembolism, further worsening respiratory failure $[5,6]$. Patients with refractory respiratory failure and ARDS normally require orotracheal intubation and invasive 
mechanical ventilation, but during COVID-19 outbreaks various European groups have adopted a different strategy using noninvasive respiratory support (NIRS), especially with the modality of continuous positive airway pressure (CPAP), either as a definitive therapeutic alternative or as a bridge for orotracheal intubation due to the lack of intensive care beds [7-9]. This was due, at least in part, to the fact that healthcare resources for critically ill patients were insufficient during the most acute phases of the pandemic in many countries.

There are several approaches and controversies regarding the management of patients with ARDS secondary to COVID-19 pneumonia. Early mechanical ventilation, which in other pathologies is considered mandatory, has been widely debated as some of the expert's early reports from Italy suggested the existence of different patient phenotypes based on different physiological parameters [10]. Due to the exponential growth of cases requiring intensive care, the health collapse has been evident in many countries. In Barcelona, for instance, during the first pandemic wave (March and April 2020), we faced a dramatic situation with a massive influx of patients in a very short time. A total of more than 2,000 patients with COVID-19 were hospitalized at our center, and more than 150 were admitted to the ICU. The big problem was that practically all the Intensive Care Unit beds were occupied very early by patients with invasive mechanical ventilation, while a relatively high percentage of critically ill patients remained under clinical surveillance outside this unit. Together with the intensive care team, it was decided that pulmonologists would make the first evaluation and initiate treatment of all those critically ill patients. Most of them showed severe desaturation and/or respiratory distress despite the use of a reservoir mask (FIO2 around 85\%). Based on initial published evidence [7-9], and the fact that many of these patients initially presented elevated spontaneous inspiratory drive with relatively large tidal volumes, and in trying to decrease the possibility of a self-inflicted lung injury (SILI) we decided to manage them in semicritical and even conventional COVID-19 units, initiating NIRS with CPAP, two-level positive airway pressure (BiPAP), or high flow through nasal cannula (HFNC) as the initial approach for severe cases. As other groups worldwide, we rapidly detected that patients with CPAP and supplemental oxygen therapy responded correctly, with orotracheal intubation being avoided in most of them [7-9]. In all cases, patients were constantly evaluated to decide if they needed to be transferred to the intensive care unit for invasive mechanical ventilation. These preliminary results confirm our approach and demonstrate that many patients could avoid orotracheal intubation if NIRS is initiated and managed by respiratory medicine and/or intensive care specialists well-trained in the use of these techniques [9]. For this reason, we have decided to prioritize this approach to treat COVID-19 patients with severe respiratory failure. Moreover, solid evidence has progressively emerged supporting the use of
CPAP for these patients. In this regard, Radovanovic et al. [7] found that the application of CPAP with the helmet system can be a valid approach for pulmonary support if used in an adequate setting, even with simple monitoring tools. This strategy could optimize the recruitment of unventilated lung regions and improve hypoxemia [7]. Similar conclusions were recently provided by Oranger et al. [8] who conclude that CPAP is feasible in severe COVID-19 patients, with intubation becoming unnecessary, and the patients can also be managed outside a standard critical care setting [8]. Although initial reports published during the early phases of the pandemics suggested that NIRS should be avoided in COVID-19 patients because of the high potential for particle aerosolization and potential contagion of healthcare personnel, the evidence has shown that the risk is low if appropriate precautions are taken. Therefore, the use of CPAP, BiPAP or HFNC is now considered safe $[11,12]$.

While CPAP is considered as the best option for most patients, BiPAP is especially recommended in those with previous thoracic diseases and/or chronic hypercapnia [13]. Since patients with severe respiratory failure caused by SARS-CoV-2 can present multiple clinical manifestations, it is also mandatory to assess many different factors to choose the most appropriate management. These factors include global prognosis based on patient own characteristics (quality of life, comorbidities), severity biomarkers based on the host inflammatory response, the response to oxygen therapy with conventional systems and the time since the onset of symptoms, among others. This careful assessment can help healthcare professionals in selecting which patients could benefit from an initial management outside the ICU despite requiring NIRS. Gatinnoni et al. [10,14], made an interesting non-invasive approach for characterizing the profile of COVID-19 patients with severe respiratory failure and suggested the most convenient respiratory support for each case. They concluded that by using chest computed tomography to approximate lung weight, two different phenotypes can be defined: the L one, characterized by low elastance - high compliance, low ventilation/perfusion ratio (VA/Q), and reduced alveolar recruitment, and the $\mathrm{H}$ phenotype, characterized by high elastance- low compliance, high right-to-left shunt and good recruitment response. This is an interesting approach because it provides relevant information when the measurement of esophageal inspiratory pressure is not possible. Measuring the esophageal pressure is the standard procedure to estimate the pleural pressure in order to have reliable information on respiratory mechanics and select the proper respiratory treatment. It is important, however, to keep in mind that these phenotypes are not static since they can change in both directions during the course of the disease. Taking advantage of all these experiences, we believe that the use of NIRS could help in organizing many hospitals in case we face further waves of SARS-CoV2 infection, prioritizing 
those semi-critical units where large numbers of patients can be treated with NIRS and careful monitoring. However, patients who can benefit from NIRS should be carefully selected, their initial response to this treatment rigorously evaluated, and, above all, delay to intubation, if necessary, should be avoided. This approach could contribute to decongest the intensive care units since these patients only require relatively simple monitoring and follow-up in adapted semi-critical units.

\section{Conclusion}

Even though more and larger studies are required, there is currently enough evidence to support the initial use of NIRS (and especially CPAP) in many patients with severe respiratory failure refractory to conventional oxygen therapy. This should be specially recommended mainly during massive COVID-19 outbreaks where patient care resources become insufficient. We consider that a careful selection of patients and the use of these techniques in expert hands can save lives and prevent orotracheal intubation in a significant number of cases. For this reason, we suggest that intermediate care units, where specialized respiratory support can be provided, should be implemented.

\section{References}

1. Zhu N, Zhang D, Wang W, Li X, Yang B, et al. (2020) A novel coronavirus from patients with pneumonia in China, 2019. N Engl J Med 382(8): 727 733.

2. (2020) World Health Organization.

3. Guan WJ, Ni ZY, Hu Y, Liang WH, Ou CQ, et al. (2020) Clinical Characteristics of Coronavirus Disease 2019 in China. N Engl J Med 382: 1708-1720.

4. ARDS Definition Task Force, Ranieri VM, Rubenfeld GD, B Taylor Thompson, Niall D Ferguson, et al. (2012) Acute respiratory distress syndrome: the Berlin Definition. JAMA 307(23): 2526-2533.
5. Siddiqi HK, Mehra MR (2020) COVID-19 illness in native and immunosuppressed states: A clinical-therapeutic staging proposal. J Heart Lung Transplant 39(5): 405-407.

6. Middeldorp S, Coppens M, Van Haaps TF, Foppen M, Vlaar AP, et al. (2020) Incidence of venous thromboembolism in hospitalized patients with COVID-19. J Thromb Haemost 18(8): 1995-2002.

7. Radovanovic D, Rizzi M, Pini S, Saad M, Chiumello DA, et al. (2020) Helmet CPAP to Treat Acute Hypoxemic Respiratory Failure in Patients with COVID-19: A Management Strategy Proposal. J Clin Med 9(4): 1191.

8. Oranger M, Gonzalez-Bermejo J, Dacosta-Noble P, Llontop C, Guerder A, et al. (2020) Continuous positive airway pressure to avoid intubation in SARS-CoV-2 pneumonia: a two period retrospective case-control study. Eur Respir J 56(2).

9. Martinez-Llorens J, Pascual-Guardia S, Aguilar R, Alvarado M, Arita M, et al. (2020) Non-invasive respiratory support in patients with COVID-19 severe respiratory failure: let's leave either nihilism or excessive invasivity. Arch Bronconeumol 73(4): 359-360.

10. Gattinoni L, Chiumello D, Caironi P, Busana M, Romitti F, et al. COVID-19 pneumonia: different respiratory treatments for different phenotypes? Intensive Care Med 2020; 46(6):1099-102.

11. Ferioli M, Cisternino C, Leo V, Pisani L, Palange P (2020) Protecting healthcare workers from SARS-CoV-2 infection: practical indications. Eur Resp Rev 29(155): 200068.

12. Li J, Fink JB, Ehrmann S (2020) High-flow nasal cannula for COVID-19 patients: low risk of bio-aerosol dispersion. Eur Respir J 55(5): 2000892.

13. (2020) Guidance for the role and use of non-invasive respiratory support in adult patients with COVID -19 (confirmed or suspected).

14. Arentz M, Yim E, Klaff L, Lokhandwala S, Riedo FX, et al. (2020) Characteristics and outcomes of 21 critically ill patients with COVID-19 in Washington State. JAMA 323(16): 1612-1674. 